\title{
Terapia fotodinâmica antimicrobiana como recurso adjuvante no tratamento endodôntico em dentes infectados: análise bibliométrica e revisão de literatura
}

Antimicrobial photodynamic therapy as an adjuvant resource in endodontic treatment in infected teeth: bibliometric analysis and literature review

Terapia fotodinámica antimicrobiana como recurso adyuvante en el tratamiento endodóntico en dientes infectados: análisis bibliométrico y revisión de la literatura

\author{
Ernani Canuto FIGUEIRÊDO JÚNIOR ${ }^{1,3}$ \\ Marcielly Moreira PEREIRA ${ }^{2}$
}

Renata Correia Sotero Dália TORRES ${ }^{3}$

Eucaé Miranda MISSIAS ${ }^{3}$

Jozinete Vieira PEREIRA ${ }^{4}$

Mônica SOARES DE ALBUQUERQUE ${ }^{3,5}$

${ }^{1}$ Doutorando em Odontologia - Programa de Pós-Graduação em Odontologia, Universidade Estadual da Paraíba - UEPB, 58429-500, Campina Grande - PB, Brasil

${ }^{2}$ Cirurgiã-Dentista, especialista em Endodontia-Instituto de Odontologia da Paraíba-IOP, 58401-145, Campina Grande-PB, Brasil

3 Instituto de Odontologia da Paraíba - IOP, 58401-145, Campina Grande - PB, Brasil

${ }^{4}$ Professora do Programa de Pós-graduação em Odontologia, Universidade Estadual da Paraíba-UEPB, 58429-500, Campina Grande - PB, Brasil

${ }^{5}$ Doutoranda em Odontologia - Programa de Pós-graduação em Odontologia, Universidade de Pernambuco-UPE, 54756-220, Camaragibe - PE, Brasil

\section{Resumo}

As infecções endodônticas são caracterizadas por apresentarem uma microbiota diversificada e complexa, desempenhando um papel importante no desenvolvimento das patologias pulpares e periapicais. Entretanto, apesar de o controle dessa infecção ser o principal objetivo do tratamento endodôntico de dentes infectados, os procedimentos químico-mecânicos podem falhar na remoção dos microrganismos patogênicos no sistema de canais radiculares, sendo necessário a realização de estratégias adjuvantes para sua desinfecção. Dentre essas, a terapia fotodinâmica antimicrobiana tem se destacado como um método promissor no tratamento das infecções endodônticas. Diante disso, este estudo consiste em uma análise bibliométrica e uma revisão de literatura acerca do uso da laserterapia através da terapia fotodinâmica como estratégia adjuvante na desinfecção dos canais radiculares. O levantamento de dados deste trabalho foi realizado nas bases de dados PUBMED, Science Direct e Scielo, incluindo-se um total de 39 artigos. Assim, foram detalhados e discutidos aspectos gerais acerca das limitações encontradas nos procedimentos químico-mecânicos convencionais durante o tratamento endodôntico, destacando nesse contexto aspectos como a importância, as vantagens, o mecanismo e a eficácia da utilização da terapia fotodinâmica como recurso adjuvante no tratamento endodôntico de dentes infectados, concluindo-se que a utilização da terapia fotodinâmica representa uma estratégia promissora e eficaz para ser utilizada em associação com os protocolos de desbridamento químico-mecânico convencional visando à potencialização da desinfecção dos canais radiculares.

Descritores: Endodontia; Tratamento do Canal Radicular; Desinfecção; Terapia a Laser; Fotoquimioterapia.

\section{Abstract}

Endodontic infections are characterized by having a diverse and complex microbiota, playing an important role in the development of pulp and periapical pathologies. However, although the control of this infection is the main objective of endodontic treatment of infected teeth, chemical-mechanical procedures may fail to remove pathogenic microorganisms in the root canal system, requiring adjuvant strategies for disinfection. Among these, antimicrobial photodynamic therapy has stood out as a promising method in the treatment of endodontic infections. Therefore, this study consists of a bibliometric analysis and a literature review, about the use of laser therapy through photodynamic therapy as an adjunct strategy in the disinfection of root canals. The data collection of this paper was carried out in the PUBMED, Science Direct and Scielo databases, including a total of 39 articles. Thus, general aspects about the limitations found in conventional chemical-mechanical procedures during endodontic treatment were detailed and discussed, highlighting aspects such as the importance, advantages, mechanism and effectiveness of using photodynamic therapy as an adjunctive resource in endodontic treatment of infected teeth, concluding that the use of photodynamic therapy represents a promising and effective strategy to be used in association with conventional chemical-mechanical debridement protocols aimed at enhancing root canal disinfection

Descriptors: Endodontics; Root Canal Therapy; Disinfection; Laser Therapy; Photochemotherapy.

\section{Resumen}

Las infecciones endodónticas se caracterizan por tener una microbiota diversa y compleja, desempeñando un papel importante en el desarrollo de las patologías pulpares y periapicales. Sin embargo, aunque el control de esta infección es el objetivo principal del tratamiento endodóntico de los dientes infectados, los procedimientos químico-mecánicos pueden no eliminar los microorganismos patógenos del sistema de conductos radiculares, lo que requiere estrategias adyuvantes para su desinfección. Entre estos, la terapia fotodinámica antimicrobiana se ha destacado como un método prometedor en el tratamiento de infecciones endodóncicas. Siendo así, este estudio consiste en un análisis bibliométrico y una revisión de la literatura sobre el uso de la terapia por láser a través de la terapia fotodinámica como estrategia complementaria para desinfectar los conductos radiculares. La recolección de datos de este trabajo se realizó en las bases de datos PUBMED, Science Direct y Scielo, que incluyeron un total de 39 artículos. Así, se detallaron y discutieron aspectos generales sobre las limitaciones encontradas en los procedimientos químico-mecánicos convencionales durante el tratamiento de endodoncia, destacando aspectos como la importancia, ventajas, mecanismo y efectividad del uso de la terapia fotodinámica como recurso complementario en el tratamiento de endodoncia de dientes infectados, concluyendo que el uso de la terapia fotodinámica representa una estrategia prometedora y efectiva para ser utilizada en asociación con protocolos convencionales de desbridamiento químico-mecánico destinados a mejorar la desinfección de los conductos radiculares.

Descriptores: Endodoncia; Tratamiento del Conducto Radicular; Desinfección; Terapia por Láser; Fotoquimioterapia.

INTRODUÇÃO

$$
\text { Infecções }
$$

caracterizadas endodônticas

são e complexa ${ }^{1-4}$, incluindo tanto bactérias grampositivas quanto gram-negativas ${ }^{1-3}$ e compreendendo principalmente as anaeróbias obrigatórias $^{2,4,5}$ e algumas facultativas ${ }^{4,5}$. 
A infecção endodôntica pode ser classificada como primária ou secundária/ persistente ${ }^{2,6}$, sendo os biofilmes associados às infecções primárias caracterizados por apresentar uma microbiota polimicrobiana usualmente mista, majoritariamente composta por bactérias Gram-negativas anaeróbias. Por outro lado, as infecções secundárias geralmente possuem uma ou mais espécies, sendo a maioria delas Gram-positivas ${ }^{7,8}$.

Uma vez que a infecção microbiana desempenha um papel importante na desenvolvimento das patologias pulpares e periapicais ${ }^{8,9}$, o principal objetivo do tratamento endodôntico (TE) de dentes com canais infectados consiste no controle dessa infecção ${ }^{9}$ através do manejo de microrganismos e seus subprodutos $^{10}$ visando à eliminação ou redução das bactérias e seus produtos no sistema de canais radiculares (SCRs) $)^{2,4,11-14}$ na busca de reduzir ou interromper a progressão da periodontite apical ${ }^{13}$ ou impedir a ocorrência de reinfecção ${ }^{15}$.

O TE consiste em procedimentos químico-mecânicos (PQMs) obtidos através da instrumentação e irrigação do SCRs com soluções desinfetantes ${ }^{13}$ como hipoclorito de sódio ou gluconato de clorexidina ${ }^{2,11}$. Entretanto, esses procedimentos de desbridamento podem falhar na remoção dos microrganismos patogênicos no SCRs ${ }^{5,13}$.

Desse modo, estratégias adjuvantes são essenciais para uma desinfecção mais eficiente do SCRs ${ }^{14,16}$. Dentre essas, a terapia fotodinâmica antimicrobiana, do inglês antimicrobial photodynamic therapy (aPDT) tem se destacado como um recurso promissor no tratamento das infecções endodônticas ${ }^{5,15-18}$.

Nesse contexto, este estudo consiste em uma análise bibliométrica e uma revisão de literatura acerca do uso da laserterapia através da aPDT como estratégia adjuvante na desinfecção do SCRs, detalhando a produção científica mais recente sobre esse tema e apresentando os principais aspectos relacionados ao conceito, aplicação e importância da aPDT como recurso adjuvante no TE.

\section{MATERIAL E MÉTODO}

O levantamento de dados do presente estudo foi realizado nas bases de dados National Library of Medicine National Institutes of Health (PUBMED), Science Direct e Scielo (Scientific Eletronic Library Online) utilizando-se os descritores "Photodynamic Therapy","Root Canal Disinfection","Endodontic Treatment" e "Endodontics", além dos operadores booleanos em língua inglesa "OR" e "AND", dispostos da seguinte forma: "(((Photodynamic Therapy) AND Root Canal Disinfection) AND Endodontic Treatment) AND Endodontics".

Para a inclusão no universo dos artigos do presente estudo, selecionaram-se artigos científicos de pesquisa e revisão de literatura publicados nos últimos cinco anos (2016-2020) sem restrições de idioma, sendo considerados apenas os artigos que empregassem abordagens/investigações sobre 0 uso da laserterapia através da aPDT na desinfecção do SCRs. Foram excluídos os artigos que não se enquadrassem nos critérios de inclusão mencionados, a exemplo de estudos que realizassem abordagens/investigações sobre o uso da desinfecção do SCRs, bem como daqueles que realizaram a aPDT com o uso de diodos emissores de luz (LEDs) e estudos que compreendessem a associação da aPDT com outros recursos adjuvantes como dispositivos sônicos ou ultrassônicos.

A pesquisa foi realizada no mês de Março de 2020 e a seleção dos artigos consistiu em três etapas, sendo a primeira delas correspondente à pesquisa dos artigos nas bases de dados conforme os critérios anteriormente mencionados, enquanto que a segunda etapa correspondeu à triagem inicial a partir da leitura dos títulos e resumos, incluindose para a etapa seguinte correspondente à leitura completa e inclusão na amostra final apenas os artigos que obedeciam aos critérios de inclusão elegidos. O fluxograma referente às estratégias de levantamento de dados e de seleção dos artigos está esquematizado na Figura 1.

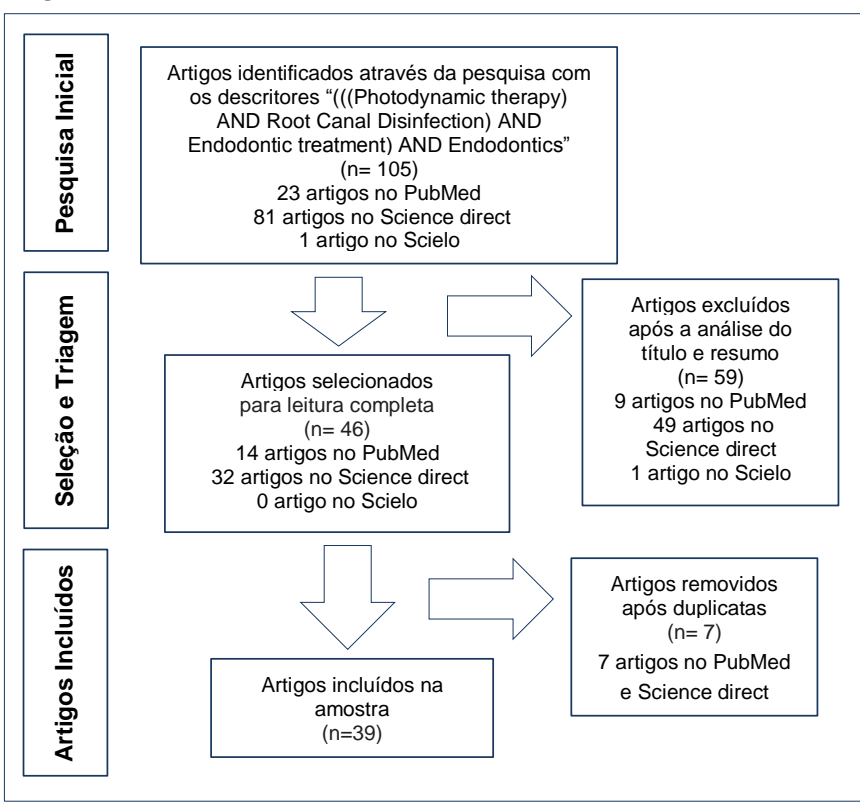

Figura 1: Fluxograma do levantamento de dados e seleção de artigos 


\section{RESULTADOS E DISCUSSÃO}

\section{Considerando-se}

os

critérios

mencionados, as pesquisas iniciais retornaram um total de 23 artigos na base de dados Pubmed, além de 81 na base de dados Science Direct e 1 artigo na base de dados Scielo. Após as etapas anteriormente mencionados de avaliação e seleção dos artigos, a amostra final de estudos incluídos na análise bibliométrica e na revisão de literatura correspondeu a um total de 39 artigos, conforme detalhados nos Quadro 1 , revisados/ discutidos a seguir sob a forma de tópicos relevantes.

Quadro 1. Descrição dos artigos selecionados e incluídos na amostra final do levantamento de dados

\begin{tabular}{|c|c|}
\hline $\begin{array}{l}\text { AUTOR/ } \\
\text { ANO }\end{array}$ & TÍTULO DO ARTIGO \\
\hline $\begin{array}{l}\text { Pourhajibagher } \\
\text { et al. } 2016^{1}\end{array}$ & $\begin{array}{l}\text { Evaluation of photo-activated disinfection effectiveness with } \\
\text { methylene blue against Porphyromonas gingivalis involved in } \\
\text { endodontic infection: An in vitro study }\end{array}$ \\
\hline $\begin{array}{l}\text { Pourhajibagher } \\
\text { et al. } 2017^{2}\end{array}$ & $\begin{array}{l}\text { The evaluation of cultivable microbiota profile in patients with } \\
\text { secondary endodontic infection before and after photo-activated } \\
\text { disinfection }\end{array}$ \\
\hline $\begin{array}{l}\text { Sarda et al, } \\
\quad 2019^{3}\end{array}$ & $\begin{array}{l}\text { Antimicrobial efficacy of photodynamic therapy, diode laser, and } \\
\text { sodium hypochlorite and their combinations on endodontic } \\
\text { pathogens }\end{array}$ \\
\hline $\begin{array}{l}\text { Pourhajibagher } \\
\text { et al. } 2020^{4}\end{array}$ & $\begin{array}{l}\text { Dual wavelengths irradiation antimicrobial photodynamic therapy } \\
\text { using indocyanine green and metformin doped with nano-curcumin } \\
\text { as an eficiente adjunctive endodontic treatment modality }\end{array}$ \\
\hline $\begin{array}{l}\text { Bolhari } \\
\text { et al,20185 }\end{array}$ & $\begin{array}{l}\text { Ex vivo assessment of synergic effect of chlorhexidine for enhancing } \\
\text { antimicrobial photodynamic therapy efficiency on expression } \\
\text { patterns of biofilm-associated genes of Enterococcus faecalis }\end{array}$ \\
\hline $\begin{array}{l}\text { Pourhajibagher } \\
\text { et al, } 2018^{6}\end{array}$ & $\begin{array}{l}\text { An experimental study for rapid detection and quantification of } \\
\text { endodontic microbiota following photo-activated disinfection via } \\
\text { new multiplex real-time PCR assay }\end{array}$ \\
\hline $\begin{array}{l}\text { Asnaashari } \\
\text { et al. } 2016^{7}\end{array}$ & $\begin{array}{l}\text { Comparison of the antibacterial effect of } 810 \mathrm{~nm} \text { diode laser and } \\
\text { photodynamic therapy in reducing the microbial flora of root canal } \\
\text { in endodontic retreatment in patients with periradicular lesions }\end{array}$ \\
\hline $\begin{array}{l}\text { Bordea } \\
\text { et al. } 2020^{8}\end{array}$ & $\begin{array}{l}\text { Evaluation the outcome of various laser therapy applications in root } \\
\text { canal disinfection: A systematic review }\end{array}$ \\
\hline $\begin{array}{l}\text { Armand } \\
\text { et al. } 2019^{9}\end{array}$ & $\begin{array}{l}\text { Comparison study of root canal disinfection by cold plasma jet and } \\
\text { photodynamic therapy }\end{array}$ \\
\hline $\begin{array}{l}\text { Akbari } \\
\text { et al. } 2017^{10}\end{array}$ & $\begin{array}{l}\text { The effect of indocyanine green loaded on a novel nano-graphene } \\
\text { oxide for high performance of photodynamic therapy against } \\
\text { Enterococcus faecalis }\end{array}$ \\
\hline $\begin{array}{l}\text { Beltes } \\
\text { et al. } 2017^{11}\end{array}$ & $\begin{array}{l}\text { Antimicrobial photodynamic therapy using indocyanine green and } \\
\text { near-infrared diode laser in reducing Entrerococcus faecalis }\end{array}$ \\
\hline $\begin{array}{l}\text { Ghorbanzadeh } \\
\text { et al. } 2018^{12}\end{array}$ & $\begin{array}{l}\text { Evaluation of the antibacterial efficacy of various root canal } \\
\text { disinfection methods against Enterococcus faecalis biofilm. An ex- } \\
\text { vivo study }\end{array}$ \\
\hline $\begin{array}{l}\text { Pourhajibagher } \\
\text { e Bahador } \\
2019^{13}\end{array}$ & $\begin{array}{l}\text { Adjunctive antimicrobial photodynamic therapy to conventional } \\
\text { chemomechanical debridement of infected root canal systems: A } \\
\text { systematic review and meta-analysis }\end{array}$ \\
\hline $\begin{array}{l}\text { Ghorbanzadeh } \\
\text { et al. } 2020^{14}\end{array}$ & $\begin{array}{l}\text { Ex Vivo comparison of antibacterial efficacy of conventional } \\
\text { chemomechanical debridement alone and in combination with light- } \\
\text { activated disinfection and laser irradiation against Enterococcus } \\
\text { Faecalis biofilm }\end{array}$ \\
\hline $\begin{array}{l}\text { Batinić } \\
\text { et al. } 2018^{15}\end{array}$ & $\begin{array}{l}\text { Comparison of final disinfection protocols using antimicrobial } \\
\text { photodynamic therapy and different irrigants after single-file } \\
\text { reciprocating instrumentation against intracanal bacterial biofilm - } \\
\text { An in vitro study }\end{array}$ \\
\hline $\begin{array}{c}\text { Rosa } \\
\text { et al. } 2017^{16}\end{array}$ & $\begin{array}{l}\text { Effectiveness of photodynamic therapy associated with irrigants } \\
\text { over two biofilm models }\end{array}$ \\
\hline $\begin{array}{l}\text { Mohammadi } \\
\text { et al. } 2017^{17}\end{array}$ & Recent advances in root canal disinfection: A review \\
\hline $\begin{array}{l}\text { Mohammadi } \\
\text { et al. } 2017^{18}\end{array}$ & Photodynamic therapy in endodontics \\
\hline $\begin{array}{l}\text { Soares } \\
\text { et al. } 2018^{19}\end{array}$ & $\begin{array}{l}\text { Exploring different photodynamic therapy parameters to optimize } \\
\text { elimination of Enterococcus faecalis in planktonic form }\end{array}$ \\
\hline $\begin{array}{l}\text { Eslami } \\
\text { et al. } 2019^{20}\end{array}$ & $\begin{array}{l}\text { The comparison of intracanal medicaments, diode laser and } \\
\text { photodynamic therapy on removing the biofilm of Enterococcus } \\
\text { faecalis and Candida albicans in the root canal system (ex-vivo } \\
\text { study) }\end{array}$ \\
\hline $\begin{array}{l}\text { Pourhajibagher } \\
\text { et al. } 2018^{21}\end{array}$ & $\begin{array}{l}\text { Exploring different photosensitizers to optimize elimination of } \\
\text { planktonic and biofilm forms of Enterococcus faecalis from infected } \\
\text { root canal during antimicrobial photodynamic therapy }\end{array}$ \\
\hline $\begin{array}{l}\text { Chiniforush } \\
\text { et al. } 2016^{22}\end{array}$ & $\begin{array}{l}\text { The in vitro effect of antimicrobial photodynamic therapy with } \\
\text { indocyanine green on Enterococcus faecalis: influence of a washing } \\
\text { vs non-washing procedure }\end{array}$ \\
\hline $\begin{array}{l}\text { Rabello } \\
\text { et al. } 2017^{23}\end{array}$ & $\begin{array}{l}\text { Does supplemental photodynamic therapy optimize the disinfection } \\
\text { of bacteria and endotoxins in one-visit and two-visit root canal } \\
\text { therapy? a randomized clinical trial }\end{array}$ \\
\hline $\begin{array}{l}\text { Plotino } \\
\text { et al.201824 }\end{array}$ & Photodynamic therapy in endodontics \\
\hline $\begin{array}{l}\text { Afkhami } \\
\text { et al.201625 }\end{array}$ & $\begin{array}{l}\text { Entrococcus faecalis elimination in root canals using silver } \\
\text { nanoparticles, photodynamic therapy, diode laser, or laser-activated } \\
\text { nanoparticles: An in vitro study }\end{array}$ \\
\hline $\begin{array}{l}\text { Mohan } \\
\text { et al.2016 }\end{array}$ & $\begin{array}{l}\text { Photoactivated disinfection (PAD) of dental root canal system- An } \\
\text { ex-vivo study }\end{array}$ \\
\hline
\end{tabular}

Quadro 1 - Continuação. Descrição dos artigos selecionados e incluídos na amostra final do levantamento de dados

\begin{tabular}{|c|c|}
\hline $\begin{array}{l}\text { AUTOR/ } \\
\text { ANO }\end{array}$ & TÍTULO DO ARTIGO \\
\hline $\begin{array}{l}\text { Pourhajibagher } \\
\text { et al.2016 }\end{array}$ & $\begin{array}{l}\text { Effects of sub-lethal doses of photo-activated disinfection against } \\
\text { Porphyromonas gingivalis for pharmaceutical treatment of } \\
\text { periodontal-endodontic lesions }\end{array}$ \\
\hline $\begin{array}{l}\text { Soares } \\
\text { et al. } 2016^{28}\end{array}$ & $\begin{array}{l}\text { Monitoring the effectiveness of photodynamic therapy with periodic } \\
\text { renewal of the photosensitizer on intracanal Enterococcus faecalis } \\
\text { biofilms }\end{array}$ \\
\hline $\begin{array}{l}\text { Susila } \\
\text { et al. } 2016^{29}\end{array}$ & $\begin{array}{l}\text { Combined effects of photodynamic therapy and irrigants in } \\
\text { disinfection of root canals }\end{array}$ \\
\hline $\begin{array}{c}\text { Garcez e } \\
\text { Hamblin } 2017^{30}\end{array}$ & $\begin{array}{l}\text { Methylene blue and hydrogen peroxide for photodynamic } \\
\text { inactivation in root canal - A new protocol for use in endodontics }\end{array}$ \\
\hline $\begin{array}{l}\text { Pourhajibagher } \\
\text { et al.2017 }\end{array}$ & $\begin{array}{l}\text { Photo-activated disinfection based on indocyanine green against cell } \\
\text { viability and biofilm formation of Porphyromonas gingivalis }\end{array}$ \\
\hline $\begin{array}{c}\text { Prażmo } \\
\text { et al. } 2017^{32}\end{array}$ & $\begin{array}{l}\text { Effectiveness of repeated photodynamic therapy in the elimination } \\
\text { of intracanal Enterococcus faecalis biofilm:an in vitro study }\end{array}$ \\
\hline $\begin{array}{l}\text { Souza } \\
\text { et al. } 2017^{33}\end{array}$ & $\begin{array}{l}\text { Evaluation of antimicrobial activity of association of chlorhexidine } \\
\text { to photosensitizer used in photodynamic therapy in root canals } \\
\text { infected by Enterococcus faecalis }\end{array}$ \\
\hline $\begin{array}{l}\text { Chiniforush } \\
\text { et al. } 2018^{34}\end{array}$ & $\begin{array}{l}\text { The effect of sublethal photodynamic therapy on the expression of } \\
\text { Enterococcalsurface protein (esp) encoding gene in Enterococcus } \\
\text { faecalis: quantitative Real-time PCR assessment }\end{array}$ \\
\hline $\begin{array}{l}\text { Golmohamadpour } \\
\text { et al,201835 }\end{array}$ & $\begin{array}{l}\text { Antimicrobial photodynamic therapy assessment of three } \\
\text { indocyanine green-loaded metal-organic frameworks against } \\
\text { Enterococcus faecalis }\end{array}$ \\
\hline $\begin{array}{c}\text { Pourhajibagher e } \\
\text { Bahador } 2018^{36}\end{array}$ & $\begin{array}{l}\text { An in vivo evaluation of microbial diversity before and after the } \\
\text { photoactivated disinfection in primary endodontic infections: } \\
\text { Traditional phenotypic and molecular approaches }\end{array}$ \\
\hline $\begin{array}{l}\text { Pourhajibagher e } \\
\text { Bahador, } 201837\end{array}$ & $\begin{array}{l}\text { Diagnostic accuracy of multiplex real-time PCR approaches in } \\
\text { compared with cultivation -based detection methods: Monitoring } \\
\text { the endopathogenic microbiota pre and post photo-activated } \\
\text { disinfection }\end{array}$ \\
\hline $\begin{array}{l}\text { Katalinić } \\
\text { et al. } 2019^{38}\end{array}$ & $\begin{array}{l}\text { The photo-activated and photo-thermal effect of the } 445 / 970 \mathrm{~nm} \\
\text { diode laser on the mixed biofilm inside root canals of human teeth in } \\
\text { vitro: A pilot study }\end{array}$ \\
\hline $\begin{array}{l}\text { Pourhajibagher } \\
\text { et al. } 2019^{39}\end{array}$ & $\begin{array}{l}\text { Antimicrobial action of photoactivated C-Phycocyanin against } \\
\text { Enterococcus faecalis biofilms: Attenuation of quorum-sensing } \\
\text { system }\end{array}$ \\
\hline
\end{tabular}

- Limitações dos PQMs na desinfecção do SCRs e a necessidade de estratégias complementares na obtenção dessa finalidade

A erradicação de microrganismos no SCRs em dentes infectados representa uma problemática constante na prática endodôntica ${ }^{8,19,20}$, uma vez que é praticamente impossível remover completamente os microrganismos em canais infectados através dos PQMs e do uso de medicação intracanal ${ }^{4,10,14}$.

Essa dificuldade é atribuída sobretudo em virtude da complexidade desse $\mathrm{SCRs}^{4,8,10,12,14,15,20}$, uma vez que em virtude da presença de ramificações, canais laterais, acessórios ${ }^{15}$, percentuais variáveis $\left(32 \%{ }^{15}\right.$; mais de $50 \%{ }^{20} ; 40 \%-60 \%{ }^{14}$ ) das paredes dentinárias no SCRs permanecem contaminadas com microrganismos e/ou seus subprodutos, além de detritos após os PQMs convencionais ${ }^{14,15,20}$.

Assim, uma vez que microrganismos residuais ainda podem ser encontrados no SCRs mesmo após a realização dos $\mathrm{PQMs}{ }^{2,9,19}$ ,e considerando-se que sua presença pode ser responsável pelo estabelecimento da reinfecção do SCRs, causando infecções endodônticas secundárias/persistentes ${ }^{2,19,21}$ e impedindo a reparação dos tecidos periradiculares ${ }^{19}$, indicase a necessidade da realização de estratégias complementares de desinfecção do SCRs ${ }^{1,13,15,16,19}$.

Dentre essas novas técnicas, destaca-se a irrigação ativada por dispositivos sônicos ${ }^{15}$ ou ultrassônicos $^{9,12,15}$, além do uso de diferentes 
tipos de lasers e da aPDT ${ }^{1,2,5,9,10-13,15}$, sendo esta última uma técnica promissora e eficaz que vem sendo cada vez mais utilizada para a desinfecção do SCRs no TE $\mathrm{T}^{2,9,10,11,14-16}$, constituindo objetivo de discussão no presente trabalho.

- Vantagens da utilização da aPDT como estratégia complementar na desinfecção do SCRs

A aplicação de lasers no TE através da realização da aPDT tem consistido em uma estratégia adjuvante na erradicação de microrganismos no SCRs ${ }^{1,3,4,11}$, em virtude do seu amplo espectro atividade antimicrobiana ${ }^{11}$.

Nesse contexto, a realização da aPDT é considerado um método minimamente invasivo no tratamento da infecção endodôntica ${ }^{21}$, apresentando vantagens como rapidez e eficácia ${ }^{19}$. Além disso, destaca-se que a mesma é capaz de inativar microrganismos patogênicos sem produzir efeitos citotóxicos, nem afetar viabilidade das células do hospedeiro, além de não gerar aumentos de temperatura ou dano térmico à dentina ou aos tecidos periodontais $^{14,19}$. Outro aspecto importante relacionado à aPDT está associado ao fato de que a mesma não induz a ocorrência de resistência microbiana ${ }^{3,13,19}$. Desse modo, esses diferentes achados da literatura corroboram as vantagens e benefícios relacionadas à utilização da aPDT durante o TE em dentes infectados.

\section{- Conceituação e mecanismos da aPDT}

Os diferentes trabalhos da literatura são concordantes no que se refere ao conceito e aos mecanismos antimicrobianos da aPDT. Nesse contexto, a aPDT consiste na utilização de uma fonte de luz que, a partir de um comprimento de onda específico, ativa um corante fotoativo não tóxico denominado fotossensibilizador (FS) frente à presença de oxigênio. Diante disso, ocorre a transferência de energia da fonte de luz ao FS, o qual uma vez excitado e na presença de oxigênio disponível, transfere-Ihe energia, dando origem à formação de espécies reativas de oxigênio (EROs) como oxigênio singleto e radicais livres ${ }^{1-5,8-11,13,15,16,19,20,21,24}$.

Desse modo, uma vez que tais produtos são citotóxicos, ocorre uma cascata de efeitos oxidantes $^{2,5,16}$ causando danos irreversíveis a diferentes moléculas da célula bacteriana como proteínas, membranas lipídicas e ácidos nucléicos ${ }^{1,4,5,9-11,15,16,20}$, levando à morte dos microrganismos ${ }^{1-4,9,11,16,20,21}$.

\section{- Fotossensibilizadores utilizados na aPDT}

Diversos fotossensibilizadores (FSs), de origem natural ou sintética, com diferentes comprimentos de onda da luz são empregados durante a aPDT ${ }^{13,21}$.

Dentre os agentes FSs comumente empregados na aPDT, cita-se o azul de metileno (AM), azul de toluidina (AT) e indocianina verde $(\mathrm{ICV})^{13,22}$. Além disso, a curcumina também tem sido mencionada e investigada como uma opção de FSs empregados durante a aPDT ${ }^{4,21}$. No entanto, dentre esses diferentes FSs, Bordea et $\mathrm{al}^{8}{ }^{8} \mathrm{e}$ Beltes et al. ${ }^{11}$ citam que o AM e o AT são os mais comumente utilizados. Esse dado também foi corroborado no presente estudo, sendo também esses os FSs empregados na maioria dos artigos incluídos na análise bibliométrica, com destaque sobretudo para o AM. A seguir, são feitas considerações acerca de cada um dos FSs relatados na literatura e aqui apresentados.

- Azul de metileno (AM)

O AM é um corante catiônico básico, de baixo peso molecular pertencente à família das fenotiazinas ${ }^{1,3,21}$, sendo 0 primeiro corante fenotiazínico sintetizado ${ }^{1}$.

O AM é comumente utilizado na aPDT em virtude de sua forte absorção no espectro de luz vermelha ${ }^{1,11}$, sendo capaz de absorver luz em comprimentos de onda de $660 \mathrm{~nm}^{21}$ a $665 \mathrm{~nm}^{11}$.

De acordo com autores como Pourhajibagher et al. ${ }^{1}$ e Pourhajibagher et al. ${ }^{21}$, esse corante mostra-se bactericida sobre bactérias Gram-positivas e Gram-negativas, sendo esses efeitos antibacterianos atribuídos à sua capacidade de penetração na membrana externa microbiana através da ligação à proteínas de superfície, conforme mencionado por Pourhajibagher et al. ${ }^{21}$.

Por outro lado, graças ao caráter hidrofílico associado ao seu baixo peso molecular e à carga positiva do AM, o mesmo interage com a molécula aniônica do lipopolissacarídeo, utilizando os canais de proteína porina na membrana externa de bactérias Gram-negativas, conforme Pourhajibagher et al. ${ }^{1}$.

- Azul de toluidina (AT)

O AT também é um corante catiônico ${ }^{2,21}$ de baixo peso molecular ${ }^{21}$, pertencente à família das fenotiazinas e assim como o AM, tem forte absorção do espectro de luz vermelha, com pico de absorção entre $634 \mathrm{~nm}^{11}$ e $635 \mathrm{~nm}^{21}$.

De acordo com Pourhajibagher et al. $^{2} \mathrm{e}$ Pourhajibagher et al. $^{21}$, o AT também mostra efeito bactericida sobre bactérias Grampositivas e Gram-negativas ${ }^{2,21}$, o qual ocorre em razão de sua penetração na membrana externa através da ligação à proteínas de superfície nas células microbianas ${ }^{21}$. 
- Indocianina verde (ICV)

ICV é um corante aniônico ${ }^{4,11,21}$ hidrossolúvel de cianina ${ }^{11}$ ativado pela luz na região do espectro infravermelho $(800-810 n m)^{5,10,11}$. Embora seja aprovado pelo Food and Drug Administração (FDA) $)^{4,10,11}$, apresentando relato de eficácia contra bactérias Gram-positivas e Gram-negativas ${ }^{10}$, autores como Pourhajibagher et al. ${ }^{4}$ afirmam que em virtude do corante ICV ter carga negativa e ser instável em solução aquosa, pode ocorrer uma interação fraca com a superfície da célula microbiana, resultando em baixa eficácia antimicrobiana.

Por outro lado, Beltes et al. ${ }^{11}$ destacam que em virtude de sua ativação pelo espectro infravermelho, o uso de FSs como ICV é capaz de causar maior penetração tecidual em comparação a casos em que são empregados FSs ativados pela luz do espectro vermelho como o AM e AT, agentes mais comumente empregados na $\mathrm{APDT}^{8,11}$, como já mencionado anteriormente.

- Curcumina (CUR)

A CUR, extraída do rizoma de açafrão (Curcuma longa L.) ${ }^{4}$ é caracterizada como um composto fenólico ${ }^{21}$ que também tem sido utilizada como uma outra opção de FS empregado na aPDT $^{4,21}$ com pico de absorção a $450 \mathrm{~nm}^{21}$.

Entretanto, autores como Pourhajibagher et al. $^{4}$ mencionam a existência de algumas propriedades químicas que limitam sua aplicação no aPDT, a exemplo da baixa solubilidade aquosa, rápida hidrólise e baixa biodisponibilidade. Apenas dois artigos ${ }^{4,21}$ incluídos e mencionados no presente estudo realizaram abordagens/investigações utilizando a CUR como FS. Assim, destaca-se a necessidade da realização de pesquisas adicionais utilizando esse FS.

- Parâmetros relacionados a eficácia da aPDT

A eficácia da erradicação de microrganismos na aPDT pode ser influenciada por vários fatores tais como parâmetros relacionados ao laser, aos FSs utilizados e ao meio onde a aPDT será aplicada, sendo esses aspectos relatados pelos diversos estudos aqui discutidos.

Dentre os parâmetros relacionados ao laser, destaca-se, sobretudo, o comprimento de onda ${ }^{10,23}$, uma vez que a luz deve ter comprimento de onda específico de acordo com o FSs a ser utilizado ${ }^{1}$. Entretanto, outros fatores são também importantes, tais como a dose de energia, a densidade de potência ${ }^{4,10,14,19,23}$ e tempo de irradiação do laser ${ }^{10,23}$.

Por outro lado, fatores relacionados aos
FSs tais como o tipo, a concentração $0^{1,4,10,14,23}$, a capacidade de ligação às células microbianas ${ }^{4} \mathrm{e}$ de difusão nos túbulos dentinários e no SCRs ${ }^{14}$, além do tempo de pré-irradiação ${ }^{1,4,10,24}$ são também convenientemente ponderados na literatura.

Além desses aspectos, Ghorbanzadeh et al. ${ }^{14}$ pontuam ainda um fator relacionado ao meio onde a terapia será aplicada, a exemplo da concentração de oxigênio disponível no SCRs.

- Aspectos referentes às diferenças metodológicas nos protocolos de aPDT entre os estudos da literatura

Uma vez considerados os diferentes tópicos apresentados e discutidos até aqui, é pertinente considerar que, conforme apontam autores como Beltes et al. ${ }^{11}$, Pourhajibagher e Bahador $^{13}$ e Bordea et al. ${ }^{8}$, em virtude da diversidade de protocolos investigados $e$ adotados, a existência de diferenças metodológicas nos estudos disponíveis na literatura leva a dificuldades de padronização e/ou interpretação dos resultados, como também ao estabelecimento de um protocolo padrão de aPDT $^{8}$.

Dentre esses aspectos, ressaltam-se diferenças relacionadas aos lasers, tais como o tipo da fonte de luz ${ }^{8,11}$, o comprimento de onda ${ }^{8}$, as doses de irradiação e potência ${ }^{8,11,13}$, o diâmetro da fibra óptica ${ }^{11}$ e a duração da irradiação $^{11,13}$. Além disso, em virtude da variedade de FSs disponíveis ${ }^{13}$, destacam-se ainda a existência de diferença entre os FSs utilizados $^{8}$, além de outros aspectos, tais como variações na concentração e tempo de préirradiação $^{13}$.

Dada a heterogeneidade de estudos incluídos na presente análise bibliométrica e revisão de literatura, esses aspectos referentes às diferenças metodológicas dos estudos disponíveis na literatura foram devidamente exemplificados, uma vez que abordagens/investigações de diversas naturezas como pesquisas incluindo metodologias ex vivo-5,9,12,14-16,20,25,26,28-30,32,33,35,38,39, in vivo e in vitro $^{2,6,36,37}$, in vitro $6,10,11,19,21,22,27,31,34$,ensaios clínicos randomizados ${ }^{7,23}$, além de revisões de literatura ${ }^{17,18,24}$, revisões sistemáticas ${ }^{8}$ e revisões sistemáticas com metanálise ${ }^{13}$ foram incluídas e avaliadas, evidenciando a existência de uma considerável produção científica acerca da utilização da aPDT na desinfecção do SCRs, corroborando assim a problemática anteriormente elucidada por Beltes et al. ${ }^{11}$, Pourhajibagher e Bahador ${ }^{13}$ e Bordea et al. ${ }^{8}$.

Entretanto, a despeito desses aspectos, 
estudos de destacada evidência científica, tal como a revisão sistemática com metanálise realizada por Pourhajibagher e Bahador $^{13}$ e a revisão sistemática realizada por Bordea et al. ${ }^{8}$ permitem corroborar que apesar da heterogeneidade entre os diferentes estudos disponíveis da literatura, a aPDT constitui uma importante estratégia auxiliar no TE de dentes infectados.

Nesse contexto, Bordea et al. ${ }^{8}$ defendem que apesar de a total erradicação microbiana no TE em casos de canais infectados ser um objetivo ainda inalcançável, o emprego da associação de soluções irrigantes antimicrobianas e da aPDT pode fornecer resultados com efeitos sinérgicos, concluindo-se que a adoção desses procedimentos pode ser considerada uma estratégia alternativa aos métodos de desinfecção convencionalmente utilizados nos casos de infecções persistentes. Além disso, de acordo com Pourhajibagher e Bahador $^{13}$, a associação de protocolos de desbridamento químico-mecânico convencional à aPDT produz uma maior redução microbiana, concluindo-se que a realização conjunta desses procedimentos oferece melhores resultados de desbridamento no SCRs em dentes infectados.

CONCLUSÃO

Diante dos achados e dos aspectos discutidos no presente estudo, percebe-se que a aPDT tem representado um recurso promissor auxiliar no TE de dentes infectados, sendo esse aspecto corroborado através da considerável produção científica relativa ao seu uso e eficácia na desinfecção do SCRs. Nesse sentido, apresar da existência de diferenças metodológicas e variações nos protocolos entre os diferentes trabalhos da literatura, os resultados evidenciados permitem evidenciar a eficácia e corroborar a importância do uso da aPDT enquanto estratégia auxiliar na desinfecção do SCRs durante o TE em dentes infectados.

\section{REFERENNCIAS}

1. Pourhajibagher $M$, Chiniforush $N$, Raoofian R, Pourakbari B, Ghorbanzadeh R, Bazarjani F et al.. Evaluation of photo-activated disinfection effectiveness with methylene blue against Porphyromonas gingivalis involved in endodontic infection: $A n$ in vitro study. Photodiagnosis Photodyn Ther. 2016;16:132-35.

2. Pourhajibagher $M$, Ghorbanzadeh R, Parker S, Chiniforush N, Bahador A. The evaluation of cultivable microbiota profile in patients with secondary endodontic infection before and after photo-activated disinfection. Photodiagnosis Photodyn Ther. 2017;18:198-203.
3. Sarda RA, Shetty RM, Tamrakar A, Shetty SY. Antimicrobial efficacy of photodynamic therapy, diode laser, and sodium hypochlorite and their combinations on endodontic pathogens. Photodiagnosis Photodyn Ther. 2019; 28:265-72.

4. Pourhajibagher $M$, Plotino $G$, Chiniforush $N$, Bahador A. Dual wavelength irradiation antimicrobial photodynamic therapy using indocyanine green and metformin doped with nano-curcumin as an efficient adjunctive endodontic treatment modality. Photodiagnosis Photodyn Ther. 2020;29:101628.

5. Bolhari B, Pourhajibagher $M$, Bazarjani $F$, Chiniforush N, Rad MR, Pirmoazen S et al.. Ex vivo assessment of synergic effect of chlorhexidine for enhancing antimicrobial photodynamic therapy efficiency on expression patterns of biofilm-associated genes of Enterococcus faecalis. Photodiagnosis Photodyn Ther. 2018;22:227-32.

6. Pourhajibagher $M$, Raoofian R, Ghorbanzadeh $R$, Bahador A. An experimental study for rapid detection and quantification of endodontic microbiota following photo-activated disinfection via new multiplex real-time PCR assay. Photodiagnosis Photodyn Ther. 2018;21:344350.

7. Asnaashari $M$, Godiny $M$, Azari-Marhabi $S$, Tabatabaei FS, Barati M. Comparison of the Antibacterial Effect of $810 \mathrm{~nm}$ Diode Laser and Photodynamic Therapy in Reducing the Microbial Flora of Root Canal in Endodontic Retreatment in Patients With Periradicular Lesions. J Lasers Med Sci. 2016;7(2):99-104.

8. Bordea IR, Hanna R, Chiniforush N, Grădinaru E, Câmpian RS, Sîrbu A, Amaroli A, Benedicenti S. Evaluation of the outcome of various laser therapy applications in root canal disinfection: A systematic review. Photodiagnosis Photodyn Ther. 2020; 29:101611.

9. Armand A, Khani M, Asnaashari M, AliAhmadi A, Shokri B. Comparison study of root canal disinfection by cold plasma jet and photodynamic therapy. Photodiagnosis Photodyn Ther. 2019;26:327-333.

10. Akbari T, Pourhajibagher $M$, Hosseini $F$, Chiniforush N, Gholibegloo E, Khoobi M et al. The effect of indocyanine green loaded on a novel nano-graphene oxide for high performance of photodynamic therapy against Enterococcus faecalis. Photodiagnosis Photodyn Ther. 2017;20:148-153.

11.Beltes C, Sakkas H, Economides N, Papadopoulou C. Antimicrobial photodynamic therapy using Indocyanine green and nearinfrared diode laser in reducing Entrerococcus faecalis. Photodiagnosis Photodyn Ther. 2017; $17: 5-8$.

12. Ghorbanzadeh A, Fekrazad R, Bahador A, Ayar 
R, Tabatabai S, Asefi S. Evaluation of the antibacterial efficacy of various root canal disinfection methods against Enterococcus faecalis biofilm. An ex-vivo study. Photodiagnosis Photodyn Ther. 2018;24:44-51.

13. Pourhajibagher $M$, Bahador A. Adjunctive antimicrobial photodynamic therapy to conventional chemo-mechanical debridement of infected root canal systems: A systematic review and meta-analysis. Photodiagnosis Photodyn Ther. 2019;26:19-26.

14. Ghorbanzadeh A, Bahador A, Sarraf P, Ayar R, Fekrazad R, Asefi S. Ex vivo comparison of antibacterial efficacy of conventional chemomechanical debridement alone and in combination with light-activated disinfection and laser irradiation against Enterococcus faecalis biofilm. Photodiagnosis Photodyn Ther. 2020;29:101648.

15. Batinić M, Ročan M, Budimir A, Anić I, Bago I. Comparison of final disinfection protocols using antimicrobial photodynamic therapy and different irrigants after single-file reciprocating instrumentation against intracanal bacterial biofilm - An in vitro study. Photodiagnosis Photodyn Ther. 2018;24:153-57.

16. Rosa RAD, Santini MF, Figueiredo JAP, Visioli F, Pereira JR, Vivan RR, Montagner F, Só MVR. Effectiveness of photodynamic therapy associated with irrigants over two biofilm models. Photodiagnosis Photodyn Ther. 2017;20:169-74.

17. Mohammadi $Z$, Jafarzadeh $H$, Shalavi $S$, Palazzi F. Recent Advances in Root Canal Disinfection: A Review. Iran Endod J. 2017;12(4):402-6.

18. Mohammadi $Z$, Jafarzadeh $H$, Shalavi $S$, Kinoshita Jl. Photodvnamic Therapy in Endodontics. J Contemp Dent Pract. 2017;18(6):534-38.

19. Soares JA, Soares SMCS, de Jesus Tavarez RR, de Castro Rizzi C, Vaz Rodrigues SCG, Maia Filho EM et al. Exploring different photodynamic therapy parameters to optimize elimination of Enterococcus faecalis in planktonic form. Photodiagnosis Photodyn Ther. 2018;22:127-131.

20. Moradi Eslami L, Vatanpour M, Aminzadeh N, Mehrvarzfar P, Taheri S. The comparison of intracanal medicaments, diode laser and photodynamic therapy on removing the biofilm of Enterococcus faecalis and Candida albicans in the root canal system (ex-vivo study). Photodiagnosis Photodyn Ther. 2019;26:157-61.

21. Pourhajibagher $M$, Kazemian $H$, Chiniforush $N$, Hosseini N, Pourakbari B, Azizollahi A et al. Exploring different photosensitizers to optimize elimination of planktonic and biofilm forms of Enterococcus faecalis from infected root canal during antimicrobial photodynamic therapy. Photodiagnosis Photodyn Ther. 2018;24:206-11.
22. Chiniforush N, Pourhajibagher $M$, Parker $S$, Shahabi S, Bahador $A$. The in vitro effect of antimicrobial photodynamic therapy with indocyanine green on Enterococcus faecalis: Influence of a washing vs non-washing procedure. Photodiagnosis Photodyn Ther. 2016;16:119-23.

23. Rabello DGD, Corazza BJM, Ferreira LL, Santamaria MP, Gomes APM, Martinho FC. Does supplemental photodynamic therapy optimize the disinfection of bacteria and endotoxins in one-visit and two-visit root canal therapy? A randomized clinical trial. Photodiagnosis Photodyn Ther. 2017;19:205-11.

24. Plotino G, Grande NM, Mercade M. Photodynamic therapy in endodontics. Int Endod J. 2019;52(6):760-74.

25. Afkhami F, Akbari S, Chiniforush N. Entrococcus faecalis Elimination in Root Canals Using Silver Nanoparticles, Photodynamic Therapy, Diode Laser, or Laser-activated Nanoparticles: An In Vitro Study. J Endod. 2017;43(2):279-82.

26. Mohan D, Maruthingal S, Indira R, Divakar DD, Al Kheraif AA, Ramakrishnaiah $R$ et al. Photoactivated disinfection (PAD) of dental root canal system - An ex-vivo study. Saudi J Biol Sci. 2016;23(1):122-27.

27. Pourhajibagher $M$, Chiniforush $N$, Raoofian $R$, Ghorbanzadeh R, Shahabi S, Bahador A. Effects of sub-lethal doses of photo-activated disinfection against Porphyromonas gingivalis for pharmaceutical treatment of periodontalendodontic lesions. Photodiagnosis Photodyn Ther. 2016;16:50-53.

28. Soares JA, Santos Soares SMC, Santos César $C A$, de Carvalho MAR, Brito-Júnior $M$, de Sousa GR, Soares BM, de Macêdo Farias L. Monitoring the effectiveness of photodynamic therapy with periodic renewal of the photosensitizer on intracanal Enterococcus faecalis biofilms. Photodiagnosis Photodyn Ther. 2016;13:123-27.

29. Susila AV, Sugumar R, Chandana CS, Subbarao CV. Combined effects of photodynamic therapy and irrigants in disinfection of root canals. J Biophotonics. 2016;9(6):603-9.

30. Garcez AS, Hamblin MR. Methylene Blue and Hydrogen Peroxide for Photodynamic Inactivation in Root Canal - A New Protocol for Use in Endodontics. Eur Endod J. 2017;2(1):1-7.

31.Pourhajibagher $M$, Chiniforush N, Ghorbanzadeh R, Bahador A. Photo-activated disinfection based on indocyanine green against cell viability and biofilm formation of Porphyromonas gingivalis. Photodiagnosis Photodyn Ther. 2017;17:61-4.

32. Prażmo EJ, Godlewska RA, Mielczarek AB. Effectiveness of repeated photodynamic 
therapy in the elimination of intracanal Enterococcus faecalis biofilm: an in vitro study. Lasers Med Sci. 2017;32(3):655-61.

33. Souza MA, Lima G, Pazinatto B, Bischoff KF, Palhano HS, Cecchin D. Evaluation of antimicrobial activity of association of chlorhexidine to photosensitizer used in photodynamic therapy in root canals infected by Enterococcus faecalis. Photodiagnosis Photodyn Ther. 2017;19:170-74.

34. Chiniforush N, Pourhajibagher M, Parker S, Benedicenti S, Shahabi S, Bahador A. The effect of sublethal photodynamic therapy on the expression of Enterococcal surface protein (esp) encoding gene in Enterococcus faecalis: Quantitative real-time PCR assessment. Photodiagnosis Photodyn Ther. 2018;24:311-17.

35. Golmohamadpour A, Bahramian B, Khoobi M, Pourhajibagher M, Barikani HR, Bahador A. Antimicrobial photodynamic therapy assessment of three indocyanine green-loaded metal-organic frameworks against Enterococcus faecalis. Photodiagnosis Photodyn Ther. 2018;23:331-38.

36. Pourhajibagher $M$, Bahador $A$. An in vivo evaluation of microbial diversity before and after the photo-activated disinfection in primary endodontic infections: Traditional phenotypic and molecular approaches. Photodiagnosis Photodyn Ther. 2018;22:19-25.

37. Pourhajibagher $M$, Bahador A. Diagnostic accuracy of multiplex real-time PCR approaches compared with cultivation -based detection methods: Monitoring the endopathogenic microbiota pre and post photoactivated disinfection. Photodiagnosis Photodyn Ther. 2018;22:140-46.

38. Katalinić I, Budimir A, Bošnjak Z, Jakovljević S, Anić I. The photo-activated and photo-thermal effect of the $445 / 970 \mathrm{~nm}$ diode laser on the mixed biofilm inside root canals of human teeth in vitro: A pilot study. Photodiagnosis Photodyn Ther. 2019;26:277-83.

39. Pourhajibagher M, Chiniforush N, Bahador A. Antimicrobial action of photoactivated CPhycocyanin against Enterococcus faecalis biofilms: Attenuation of quorum-sensing system. Photodiagnosis Photodyn Ther. 2019;28:286-91.

\section{CONFLITO DE INTERESSES}

Os autores declaram não haver conflitos de interesse

\section{AUTOR PARA CORRESPONDÊNCIA}

Ernani Canuto Figueirêdo Júnior

Universidade Estadual da Paraíba - UEPB

Departamento de Odontologia.

Rua das Baraúnas, 351, Bairro Universitário

58429-500 Campina Grande-PB, Brasil.

+ 55 (83) 3315-3300

E-mail: ernanicfjunior@outlook.com
Submetido em 06/10/2020

Aceito em 26/01/2021 